\title{
Silencing of periostin inhibits nicotine-mediated tumor cell growth and epithelial-mesenchymal transition in lung cancer cells
}

\author{
SHU-QIANG WU*, YA-ER LV*, BAI-HUA LIN, LI-MIN LUO, SHI-LIANG LV, AI-HONG BI and YONG-SHI JIA \\ Department of Radiation Oncology, Zhejiang Provincial People's Hospital, Hangzhou, Zhejiang 310014, P.R. China
}

Received May 25, 2012; Accepted October 18, 2012

DOI: $10.3892 / \mathrm{mmr} .2013 .1267$

\begin{abstract}
Nicotine has been found to induce the proliferation of lung cancer cells through tumor invasion and to confer resistance to apoptosis. Periostin is abnormally highly expressed in lung cancer and is correlated with angiogenesis, invasion and metastasis. Here, we investigated the roles of periostin in the lung cancer cell proliferation, drug resistance, invasion and epithelial-mesenchymal transition (EMT) induced by nicotine. The periostin gene was silenced using small interfering RNA (siRNA) in A549 non-small cell lung cancer (NSCLC) cells. The cells were transfected with control or periostin siRNA plasmids. Periostin mRNA was evaluated by quantitative reverse transcription-polymerase chain reaction (RT-PCR). Cell proliferation was detected using the MTT assay and cell apoptosis was detected by Annexin V-FITC and propidium iodide (PI) double staining. Tumor invasion was detected by the Boyden chamber invasion assay. Western blotting was performed to detect the expression of the EMT marker Snail. Our results revealed that stably periostin-silenced cells were acquired by G418 screening, and the periostin mRNA expression levels of which were decreased by nearly $80 \%$. Periostin-silenced A549 cells exhibited reduced cell proliferation, elevated sensitivity to chemotherapy with cisplatin, decreased cell invasion and Snail expression $(\mathrm{P}<0.05)$. Nicotine upregulated the periostin protein levels in the A549 cells and this upregulation was not blocked by the generalized nicotinic acetylcholine receptor (nAChR) antagonist, hexamethonium. In conclusion, periostin is one of the targets regulated by nicotine in lung cancer cells and is involved in the cancer cell growth, drug resistance, invasion and EMT induced by nicotine.
\end{abstract}

Correspondence to: Dr Shu-Qiang Wu, Department of Radiation Oncology, Zhejiang Provincial People's Hospital, 158 Shangtang Road, Hangzhou, Zhejiang 310014, P.R. China

E-mail: wusqzj@163.com

*Contributed equally

Key words: periostin, nicotine, lung cancer, epithelial-mesenchymal transition, siRNA, chemoresistance, migration

\section{Introduction}

Periostin, also known as osteoblast-specific factor 2 (OSF-2), is an adhesion molecule that was initially identified in mouse osteoblastic cells as a secreted extracellular matrix (ECM) protein (1). Periostin may interact with other ECM proteins and induce cell adhesion and motility by binding to integrins (2). Periostin is highly expressed in many normal connective tissues, including periodontal ligaments, the fascia of muscles and joint ligaments, and may be involved in structural maintenance and tissue development (3).

Abnormally high expression levels of periostin protein in a variety of human tumors, including thyroid cancer, oral cancer, gastric cancer and breast cancer, have been reported (4-7). Furthermore, periostin overexpression correlates with the angiogenesis, invasion and metastasis of these tumors. Periostin also stimulates tumor cell growth by promoting cell survival and angiogenesis through the Akt/PKB pathway $(7,8)$. As a mesenchymal-specific protein, periostin is expressed and secreted by tumor mesenchymal cells. Overexpression of the periostin gene in 293T cells has been reported to cause epithelial-mesenchymal transition (EMT) as well as cell migration, invasion and adhesion (9). Moreover, these periostin-overexpressing recombinant cells quickly formed metastatic loci when transplanted into immune-deficient mice (9).

Lung cancer is a malignant tumor with high occurrence that originates from normal bronchial epithelial cells. The majority of lung cancers are non-small cell lung cancer (NSCLC), and patients with NSCLC are most commonly detected at an advanced stage, leading to a low 5-year survival rate $(\sim 15 \%)$. Cigarette smoking has been implicated in the pathogenesis and increased metastasis of lung cancer (10). Nicotine is the most active carcinogen in cigarettes and may induce cell proliferation, invasion and EMT in breast and lung cancer cells (11).

Although the stimulation by nicotine of the proliferation, invasion and EMT of NSCLC has been confirmed by a number of studies, the relationship between nicotine and periostin has not been reported. Furthermore, the overexpression of periostin has been found in the cells and serum of NSCLC and is related to the proliferation and migration of tumor cells and the poor survival of patients $(12,13)$. Therefore, we propose periostin to be a novel therapeutic target for NSCLC. We investigated the effects on the proliferation, drug resistance, invasion and EMT of NSCLC cells of silencing periostin with small interfering RNA (siRNA). Our study indicated that peri- 
ostin may be a downstream target gene regulated by nicotine in the NSCLC cells.

\section{Materials and methods}

Cell culture. The human lung cancer cell line A549 was purchased from the Institute of Biochemistry and Cell Biology, Shanghai Institutes for Biological Sciences, Chinese Academy of Sciences. The cells were cultured in DMEM media (Invitrogen-Gibco, Carlsbad, CA, USA) supplemented with $10 \%$ fetal bovine serum (FBS; Sijichun Bioengineering Materials Inc., Hangzhou, Zhejiang, China), $100 \mathrm{U} / \mathrm{ml}$ penicillin and $100 \mu \mathrm{g} / \mathrm{ml}$ streptomycin, in a humidified incubator at $37^{\circ} \mathrm{C}$ with $5 \% \mathrm{CO}_{2}$. The cells were seeded in 24 -well culture plates at a density of $1 \times 10^{6} / \mathrm{ml}$ and treated with $1 \mu \mathrm{M}$ nicotine (Sigma Chemical Co., St. Louis, MO, USA). In studies using the generalized nicotinic acetylcholine receptor (nAChR) antagonist hexamethonium, quiescence of the cells was induced by serum starvation for $24 \mathrm{~h}$, followed by treatment with nicotine.

Construction of and transfection with periostin siRNA plasmid. The complementary oligonucleotides for periostin siRNA were obtained from the GenBank (accession number NM_006475.2) and were designed according to the general designing principles of siRNA. Two-step PCR was performed to amplify siRNA expression cassettes using forward primers for U6 promoter amplification and reverse primers (at 136, 246, $268 \mathrm{bp}$ ) for periostin at $94^{\circ} \mathrm{C}$ for $30 \mathrm{sec}$ and $72^{\circ} \mathrm{C}$ for $90 \mathrm{sec}$ for 40 cycles. The PCR products were ligated with plasmid pRNAT-U6.1 using T4 DNA ligase at $16^{\circ} \mathrm{C}$ overnight, followed by transformation of competent Escherichia coli DH5 $\alpha$. The transformed DH5 $\alpha$ was selected by blue-white screening, and confirmed by enzyme digestion and gene sequencing (Shen You Inc., Shanghai, China). The pRNAT-U6.1-periostin plasmid with the correct sequence was amplified and purified in large quantities.

A549 cells were seeded in 6-well plates at a density of $4 \times 10^{5} / \mathrm{ml}$. After culturing for $24 \mathrm{~h}$, the cells were transfected with either pRNAT-U6.1-periostin siRNA plasmid or pRNAT-U6.1 control plasmid. Reverse transcription-polymerase chain reaction (RT-PCR) and real-time PCR were performed to detect periostin mRNA expression $48 \mathrm{~h}$ after transfection. The A549 cells with successful periostin silencing were selected for further G418 screening $(400 \mu \mathrm{g} / \mathrm{ml})$.

RT-PCR for detecting periostin mRNA expression following transfection with periostin siRNA. Total RNA was isolated from the A549 cells $48 \mathrm{~h}$ after transfection and RT-PCR was performed to quantify the periostin mRNA expression levels normalized to $\beta$-actin. cDNA was synthesized from RNA $(5 \mu \mathrm{g})$ by reverse transcription using random primers (SuperScript III First-Strand Synthesis SuperMix kit; Invitrogen, Carlsbad, CA, USA). The forward and reverse primers were synthesized by Yingjun Biotechnology, Inc. (Shanghai, China) and are as follows: periostin forward, 5'-AGGCAAACAGCTCAGAGTCTTCGT-3' and reverse, 5'-TGCAGCTTCAAGTAGGCTGAGGAA-3'; and $\beta$-actin forward, 5'-CTGGCACCACACCTTCTACAATGA-3' and reverse, 5'-TTAATGTCACGCACGATTTCCCGC-3'. The conditions for the PCR of periostin and $\beta$-actin mRNA are $94^{\circ} \mathrm{C}$ for $4 \mathrm{~min}(1 \mathrm{cycle}) ; 94^{\circ} \mathrm{C}$ for $30 \mathrm{sec}, 57^{\circ} \mathrm{C}$ for $30 \mathrm{sec}$ and $72^{\circ} \mathrm{C}$ for $1 \mathrm{~min}$ (33 cycles); and $72^{\circ} \mathrm{C}$ for $7 \mathrm{~min}$ ( 1 cycle). The PCR products were subjected to electrophoresis on a $1.5 \%$ agarose gel containing ethidium bromide and then visualized under ultraviolet light.

Real-time PCR analysis. Periostin mRNA expression levels were further analyzed to confirm the results of RT-PCR using an RT-Cycler ${ }^{\text {TM }}$ Real-Time PCR Detection System (CapitalBio, Ltd., Beijing, China) with SYBR-Green (Molecular Probes, Eugene, OR, USA). Following RNA extraction and the reverse transcription of RNA to cDNA, $1 \mu \mathrm{l}$ cDNA was added to a $20-\mu 1$ reaction mixture containing $0.5 \mathrm{X}$ SYBR-Green, $1 \mathrm{X}$ TransStart Green qPCR SuperMix (TransGen Biotech Co., Ltd., Beijing, China) and $0.5 \mu \mathrm{mol} / 1$ primer sets. The cycling conditions for the two genes were as follows: $95^{\circ} \mathrm{C}$ for $5 \mathrm{~min}$ for 1 cycle, followed by $95^{\circ} \mathrm{C}$ for $45 \mathrm{sec}, 57^{\circ} \mathrm{C}$ for $30 \mathrm{sec}$ and $72^{\circ} \mathrm{C}$ for $20 \mathrm{sec}$ for 40 cycles. The expression levels of the cDNA of the two genes were internally normalized to those of $\beta$-actin. The $2^{-\Delta \Delta C T}$ method was used to calculate the relative quantitative levels of periostin mRNA. Each experiment was performed in duplicate and repeated three times.

MTT assay. The cell growth rate was determined by MTT assay (Sigma Chemical Co.). Briefly, $100 \mu \mathrm{l}$ cells at the logarithmic growth phase were seeded into 96 -well culture plates at a density of $1 \times 10^{3}$ cells/well. Then, cells transfected with control or periostin siRNA plasmids were cultured with DMEM media supplemented with $10 \%$ FBS and incubated with $1 \mu \mathrm{M}$ nicotine (Sigma) for 1, 2, 3, 4 and 5 days. An MTT solution $(5 \mathrm{mg} / \mathrm{ml}, 10 \mu \mathrm{l})$ was added to each well and the plates were incubated at $37^{\circ} \mathrm{C}$ for $4 \mathrm{~h}$. After centrifugation at 3,000 rpm for $10 \mathrm{~min}$, the supernatant was removed and the remaining formazan pellet was dissolved completely in $100 \mu \mathrm{l}$ DMSO. An ELISA plate reader was used to measure the absorbance at $570 \mathrm{~nm}$ to determine the amount of pellet.

Cell apoptosis assay. A549 cells were cultured in vitro and randomly assigned to one of four groups: normal control (siRNA-C), periostin-silenced (siRNA-P), normal treated with nicotine (siRNA-C+N) and periostin-silenced treated with nicotine (siRNA-P+N). After $72 \mathrm{~h}, 2 \times 10^{5}$ cells from each group were harvested for centrifugation at 2,000 rpm for $5 \mathrm{~min}$. After washing with PBS buffer, the pellet was resuspended in $100 \mu \mathrm{l} 1 \mathrm{X}$ binding buffer and incubated with $2.5 \mu \mathrm{l}$ Annexin V and $5 \mu \mathrm{l}$ propidium iodine (PI) (final concentration: $10 \mu \mathrm{g} / \mathrm{ml}$ ) for $15 \mathrm{~min}$ in the dark. Apoptosis was determined by flow cytometry and analyzed using Lysis software. At least 10,000 events were analyzed for each sample.

Cell migration assay. The in vitro migration of the A549 cells was evaluated using a Boyden chamber invasion assay. A Transwell filter membrane was used for experiments in 24-well tissue culture plates. The lower side of the filter was coated with type I collagen $(0.5 \mathrm{mg} / \mathrm{ml})$ and contained low-serum medium in the presence or absence of nicotine. In the upper part of the Transwell plate, $5 \times 10^{4}$ cells transfected with control or periostin siRNA plasmid were resuspended in $100 \mu 1$ DMEM medium and plated. The cells were fixed with methanol and 
A

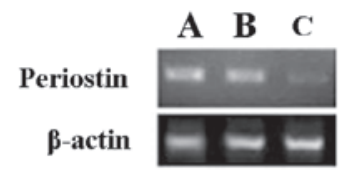

B

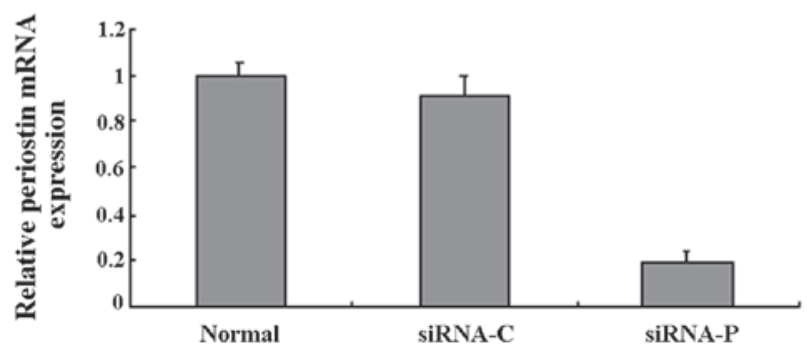

Figure 1. Efficacy of periostin siRNA transfection. (A) Periostin mRNA expression was clearly reduced by periostin siRNA, as shown by RT-PCR on agarose gel. A, Control cells without plasmid; B, Cells transfected with pRNAT-U6.1 control plasmid; C, Cells transfected with plasmid of periostinsiRNA. (B) The level of periostin mRNA after transfection with periostin siRNA plasmid was decreased by nearly $80 \%(\mathrm{P}<0.05)$. The control plasmid had no influence on the periostin expression of the A549 cells. siRNA-C, A549 cells transfected with control plasmid; siRNA-P, A549 cells transfected with periostin siRNA.

stained with hematoxylin and eosin (Sigma). After $24 \mathrm{~h}$, the cells on the upper surface of the filter were removed. The cells that had migrated to the lower part were counted by light microscopy (magnification x200) as the number of migrated cells. Each sample was assayed in triplicate and repeated twice

Western blot analysis. Proteins were extracted for the determination of their concentrations using a bicinchoninic acid protein concentration assay kit (Beijing Biosea Biotechnology Co., Ltd., Beijing, China). Following separation on sodium dodecyl sulfate-polyacrylamide gel electrophoresis (SDS-PAGE) gels (polyacrylamide concentration $100 \mathrm{~g} / \mathrm{l})$, the proteins $(50 \mu \mathrm{g})$ were electrophoretically transferred to a PVDF membrane. The PVDF membrane was blocked with $3 \%$ BSA at $37^{\circ} \mathrm{C}$ for $1 \mathrm{~h}$ and probed with the mouse monoclonal antibodies against Snail $(1: 1,000)$ or periostin (Santa Cruz Biotechnology, Santa Cruz, CA, USA). The second antibody was horseradish peroxidase-conjugated rabbit anti-mouse IgG and used at a dilution of 1:1,000 for $2 \mathrm{~h}$ at room temperature. A chemiluminescence method was used to visualize the density of the targeted bands. $\beta$-actin was used as an internal control.

Statistical analysis. Commercially available software (SPSS version 14.0) was used to perform the statistical analysis. The data are expressed as the mean \pm SD. The Student's t-test (unpaired, two-tailed) was performed to compare the means between the two groups. $\mathrm{P}<0.05$ was considered to indicate a statistically significant result.

\section{Results}

Efficacy of periostin siRNA transfection. RT-PCR was used to detect the levels of periostin mRNA following the transfection of the A549 cells with siRNA. The cells transfected with the siRNA for periostin showed clearly decreased expression of periostin mRNA. However, no difference in periostin mRNA levels was observed between the control plasmid-transfected cells and the untransfected cells (Fig. 1A). To further confirm the results of RT-PCR quantitatively, real-time RT-PCR was carried out. The periostin mRNA levels of the A549 cells which stably expressed periostin siRNA were decreased by $\sim 80 \%$ compared with those of the untransfected A549 cells, whereas the control plasmid had no influence on the periostin mRNA levels in the A549 cells (Fig. 1B). The periostin mRNA expression levels were significantly reduced by transfection with periostin siRNA. The A549 cells which had been successfully transfected with periostin siRNA were further screened in culture media supplemented with G418 (400 $\mu \mathrm{g} / \mathrm{ml})$.

Periostin siRNA inhibited nicotine-induced lung cancer cell proliferation. To investigate the effect of silencing periostin on cell proliferation, we performed an MTT assay to evaluate the growth of the A549 cells. The results indicated that the A549 cells stably expressing periostin siRNA showed significantly slower growth on days 3,4 and 5 than the normal A549 cells (Fig. 2A). We observed no difference in cell growth between the cells transfected with control plasmid and the normal A549 cells. Nicotine treatment increased the growth of the A549 cells transfected with control plasmid. The effect of nicotine was significantly reduced by transfection with periostin siRNA (Fig. 2B).

Periostin siRNA attenuated the nicotine-induced chemoresistance of lung cancer cells. A549 cells were incubated with cisplatin $(5 \mu \mathrm{g} / \mathrm{ml})$ throughout this experiment. The cells were randomly divided into four groups: siRNA-C, siRNA-P, siRNA-C+N and siRNA-P+N. Treatment with nicotine for $72 \mathrm{~h}$ significantly decreased the apoptotic rate of the A549 cells treated with cisplatin, as shown by flow cytometry. However, this chemoresistance was reversed in the periostin siRNAtransfected cells which had a significantly higher apoptotic rate than the cells transfected with the control plasmid. In the cells that did not undergo nicotine treatment, periostin siRNA transfection alone did not increase the rate of apoptosis (Fig. 2C and D).

Periostin siRNA inhibited invasion and the expression of EMT marker protein Snail. Periostin siRNA-transfected cells showed a slightly lower index of invasion than cells transfected with control plasmid. Following nicotine treatment, the A549 cells showed a significantly higher index of invasion. However, in the nicotine-treated A549 cells, siRNA-periostin transfection significantly decreased the index of invasion compared with that of cells transfected with the control plasmid (Fig. 3A).

The expression of the EMT marker Snail was determined by western blot analysis. In cells tranfected with the control plasmid (siRNA-C), the Snail protein levels were significantly increased by nicotine treatment. However, transfection with periostin siRNA significantly decreased the Snail protein expression levels in the A549 cells treated with nicotine (Fig. 3B and C).

Nicotine induced periostin upregulation in lung cancer cells in a nAChR-independent manner. Western blot analysis revealed that treatment with $1 \mu \mathrm{M}$ nicotine for $24 \mathrm{~h}$ significantly upregulated periostin protein expression levels in the 

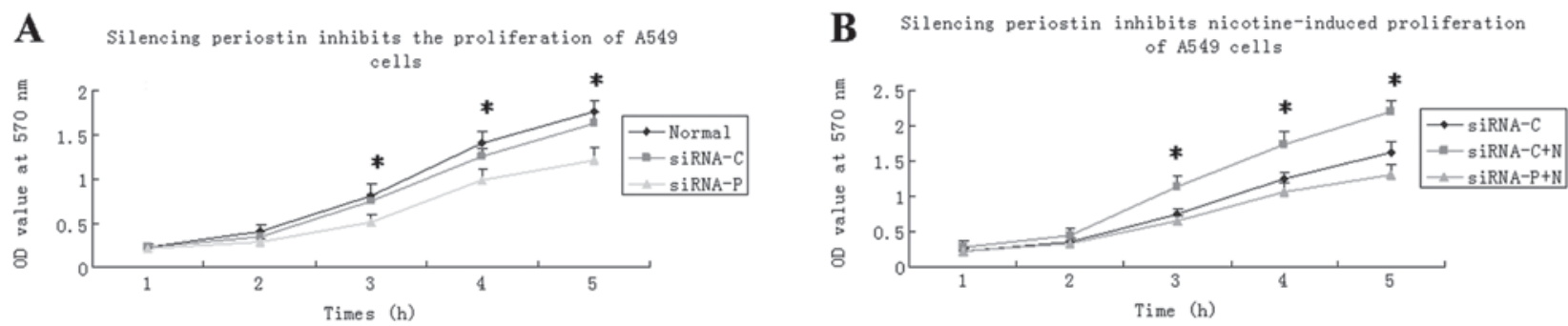

C

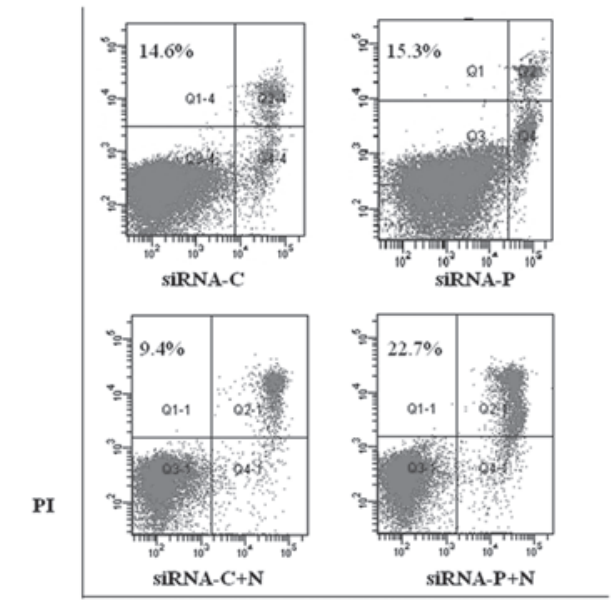

Deriostin siRNA attenuated nicotine-induced chemoresistance

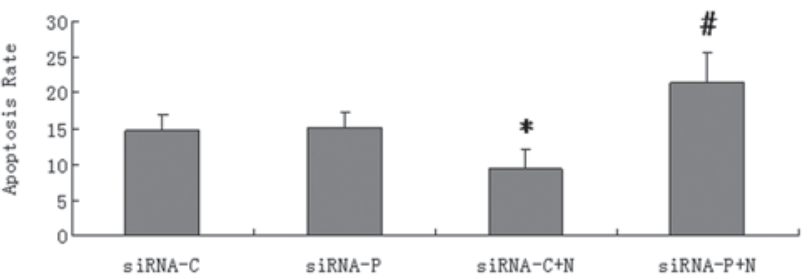

Annexin V-FITC

Figure 2. Silencing periostin inhibits the proliferation of A549 cells in vitro. (A) Growth curves of A549 cells based on data from MTT assays in normal A549 cells (normal) and A549 cells transfected with control plasmid (siRNA-C) or periostin siRNA plasmid (siRNA-P). From day 3, the stably periostin siRNA-expressing A549 cells started to grow slowly $(\mathrm{P}<0.05)$. (B) Growth curves of siRNA-C cells and A549 cells treated with nicotine and transfected with control plasmid (siRNA-C+N) or periostin siRNA plasmid (siRNA-P+N). From day 3, periostin silencing decreased the growth of the A549 cells treated with nicotine $(\mathrm{P}<0.05)$. (C) Cell apoptosis was determined by Annexin V-FITC and propidium iodide (PI) double staining using flow cytometry after $72 \mathrm{~h}$ of treatment. Periostin siRNA transfection did not increase the apoptotic rate of the A549 cells without nicotine treatment. Nicotine decreased the apoptosis rate significantly in the A549 cells $(\mathrm{P}<0.05)$ and periostin siRNA transfection significantly increased the apoptosis rate of the nicotine-treated A549 cells. Representative images from three experiments are shown. (D) Apoptotic rates are shown in A549 cells transfected with control or periostin siRNA plasmids, with or without nicotine treatment $(72 \mathrm{~h})$. Data are expressed as the mean $\pm \mathrm{SD}$ and were compared using a two-tailed, unpaired t-test. ${ }^{*} \mathrm{P}<0.05$, significant difference from the siRNA-C group. ${ }^{\#} \mathrm{P}<0.05$, significant difference from the siRNA-C+N group.
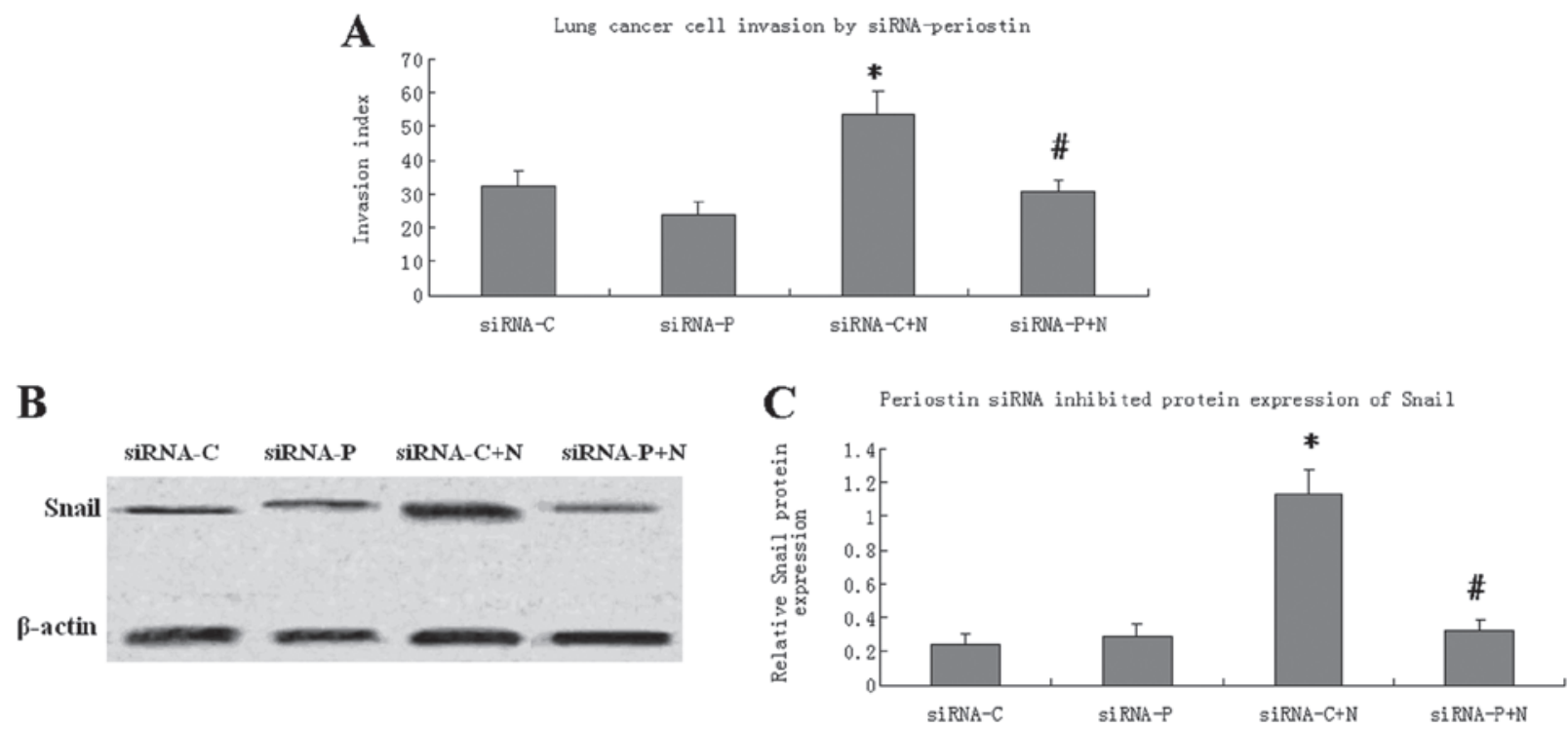

Figure 3. Periostin siRNA inhibited the cell invasion and the expression of EMT marker protein Snail induced by nicotine. (A) The pro-invasive effect of nicotine was shown in a Boyden chamber assay. A549 cells stably expressing periostin siRNA (siRNA-P) or control plasmid (siRNA-C) were treated with nicotine and investigated for cell invasion activity. (B) The inhibitory effect of periostin siRNA on nicotine-induced Snail protein expression. The siRNA-P and siRNA-C cells were treated with nicotine (to form siRNA-C+N and siRNA-P+N cells, respectively) and evaluated for protein expression of the EMT marker Snail by western blot analysis. $\beta$-actin served as a loading control. One representative image is shown from three independent experiments. (C) Relative expression of Snail by the four groups. The Y ordinate indicates the grey value of Snail normalized to that of $\beta$-actin. Data are expressed as the mean \pm SD and a two-tailed, unpaired t-test was performed. ${ }^{*} \mathrm{P}<0.05$, significant difference from the siRNA-C group. ${ }^{*} \mathrm{P}<0.05$, significant difference from the siRNA-C $+\mathrm{N}$ group. 

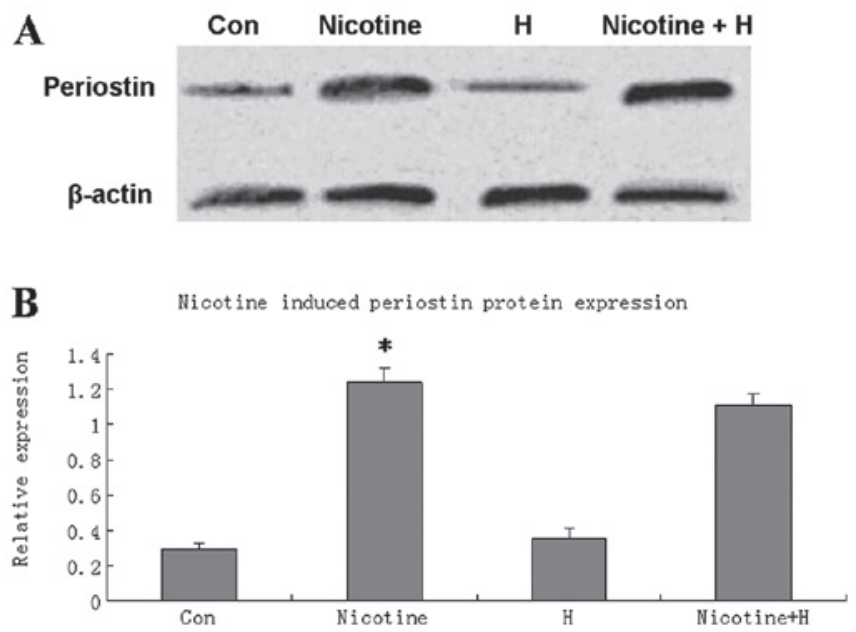

Figure 4. Nicotine induced periostin upregulation in lung cancer cells in a nAChR-independent manner. (A) The generalized nAChR antagonist hexamethonium $(20 \mu \mathrm{M})$ was applied to A549 cells for $6 \mathrm{~h}$. The A549 cells were then treated with nicotine $(1 \mu \mathrm{M})$ for $24 \mathrm{~h}$ and evaluated for periostin protein expression by western blot analysis. One representative image from three independent experiments is shown. (B) Relative expression of periostin in the four groups. The $\mathrm{Y}$ ordinate indicates the grey value of periostin normalized to that of $\beta$-actin. The relative expression of periostin was significantly higher in A549 cells following nicotine treatment. Con, A549 cells; nicotine A549 cells treated with nicotine; H, A549 cells treated with hexamethonium nicotine $+\mathrm{H}$, A549 cells treated with nicotine and hexamethonium. Data are expressed as the mean \pm SD and a two-tailed, unpaired t-test was performed ${ }^{*} \mathrm{P}<0.05$, significant difference from the control group.

A549 cells, suggesting that periostin may be a downstream target of nicotine in lung cancer cells. Pretreatment with a generalized nAChR antagonist, hexamethonium, did not influence the expression of periostin in the nicotine-treated or nicotine-untreated A549 cells (Fig. 4).

\section{Discussion}

In this study, we investigated the effect of periostin on the proliferation, drug resistance, tumor invasion and EMT of NSCLC cells by silencing periostin with RNAi. A stably periostin-silenced cell line was acquired by G418 screening. The periostin mRNA levels of the NSCLC cells with stably expressed periostin siRNA were decreased by $\sim 80 \%$ compared with those of the NSCLC cells without treatment. The periostin-silenced NSCLC cells exhibited reduced cell proliferation, elevated sensitivity to chemotherapy with cisplatin, decreased cell invasion and Snail expression levels. Nicotine upregulated periostin protein in the NSCLC cells, and this effect was not blocked by the generalized nAChR antagonist, hexamethonium.

Periostin overexpression in human tumors may promote tumor growth and survival by inducing the Akt/PKB pathway (14). However, periostin overexpression is not always associated with increased proliferation, as shown in several human cancer cell lines (15). In our study, we found that periostin mRNA was expressed in the A549 NSCLC cell line and may be fully silenced by RNAi (Fig. 1). In order to explore the effects of silencing periostin, we used an MTT assay to evaluate the proliferation of the NSCLC cells. The results revealed that the stably periostin siRNA-expressing NSCLC cells grew more slowly than the cells transfected with control plasmid (Fig. 2A). Furthermore, nicotine treatment increased the proliferation of the NSCLC cells and this effect was reversed by periostin silencing (Fig. 2B).

Our study demonstrated that periostin silencing may significantly enhance the sensitivity of NSCLC cells to the chemotherapy agent cisplatin. Cisplatin treatment induced a moderate degree of apoptosis in the NSCLC cells and nicotine increased the cell survival rate and reduced the apoptotic rate. However, periostin silencing significantly increased apoptosis in the cells treated with cisplatin and nicotine. By contrast, with nicotine treatment, periostin silencing showed little influence on the apoptotic rate of the NSCLC cells. This phenomenon may be caused by nicotine and periostin inducing common survival pathways. The activation of survival pathways by nicotine may enhance the dependence of the NSCLC cells on these pathways and increase their sensitivity to periostin silencing. Nicotine stimulates proliferation and inhibits apoptosis in colon cancer cells by the activation of survival pathways, for example, by significantly increasing the expression of PI3K and the P-Akt/Akt ratio (16). The Akt/PKB pathway also participates in the enhanced proliferation and survival promoted by periostin in several human cancer cell lines (14). The possible mechanisms underlying the shared survival pathways of nicotine and periostin require further investigation. It has been reported that nicotine may induce chemoresistance by activating anti-apoptotic pathways in several cancer cell lines (17-19), therefore the chemosensitizing effect due to shared survival pathways is a promising therapeutic approach in periostin-targeting therapy.

Tumor invasion is a complex, multi-step process that involves the alteration of cell adhesion to extracellular matrix protein interactions. Nicotine enhances the phosphorylation of calpains and increases the expression levels of COX2, VEGF and VEGFR2, which are molecules affecting the invasive process (20-22). Our findings show that the pro-invasive effects of nicotine may be reversed by periostin silencing. Given the correlation between periostin overexpression and tumor proliferation and migration, our results indicate that periostin is not only a promising biomarker for NSCLC prognosis but also a potential target for therapeutical intervention.

EMT is a vital step occurring in epithelial cells for the acquisition of a malignant phenotype, including the capabilities of migration, invasion and metastasis to a new location (23). Our results revealed that after nicotine treatment, the periostin gene-silenced NSCLC cells had significantly downregulated expression levels of Snail, which is an EMT-inducing gene. This suggests that periostin expression is required for EMT to occur in NSCLC cells.

Our study revealed that periostin expression was regulated by nicotine in NSCLC cells, suggesting that periostin is a downstream target of nicotine. The pathophysiological effects of nicotine are mediated by $\mathrm{nAChRs,} \mathrm{which} \mathrm{are} \mathrm{mainly}$ expressed on neurons and neuromuscular junctions (24). $\mathrm{nAChRs}$ have also been detected in primary endothelial cells as well as the A549 human NSCLC cell line. Nicotine has been shown to induce the invasion and migration of NSCLC cells in a $\alpha 7$-receptor-dependent manner (11). In our study, we pretreated A549 cells with a generalized nAChR antagonist hexamethonium, followed by nicotine treatment. 
In the nicotine-induced A549 cells, the elevated expression of periostin was not affected by hexamethonium. This indicates that nicotine may upregulate periostin expression through nAChR-independent pathways. It also suggests that periostin overexpression is essential but not sufficient for nicotine-induced proliferation, drug resistance, invasion and EMT in NSCLC cells.

In conclusion, we identified that periostin is a nicotine-regulated gene and contributes to nicotine-induced cell growth, drug resistance, tumor invasion and EMT in NSCLC cells. Silencing periostin expression using siRNA may inhibit the proliferation, chemoresistance, invasion and EMT of NSCLC cells induced by nicotine. Periostin is a nicotine-regulated gene in NSCLC cells. Therefore, periostin may be a promising therapeutical target for NSCLC intervention in the future.

\section{References}

1. Takeshita S, Kikuno R, Tezuka K and Amann E: Osteoblast specific factor 2: cloning of a putative bone adhesion protein with homology with the insect protein fasciclin I. Biochem J 294 271-278, 1993

2. Gillan L, Matei D, Fishman DA, Gerbin CS, Karlan BY and Chang DD: Periostin secreted by epithelial ovarian carcinoma is a ligand for alpha(V)beta(3) and alpha(V)beta(5) integrins and promotes cell motility. Cancer Res 62: 5358-5364, 2002.

3. Hamilton DW: Functional role of periostin in development and wound repair: implications for connective tissue disease. J Cell Commun Signal 2: 9-17, 2008.

4. Puppin C, Fabbro D, Dima M, Di Loreto C, Puxeddu E, Filetti S, Russo D and Damante G: High periostin expression correlates with aggressiveness in papillary thyroid carcinomas. J Endocrinol 197: 401-408, 2008.

5. Zhang Y, Zhang G, Li J, Tao Q and Tang W: The expression analysis of periostin in human breast cancer. J Surg Res 160: 102-106, 2010.

6. Li JS, Sun GW, Wei XY and Tang WH: Expression of periostin and its clinicopathological relevance in gastric cancer. World $\mathrm{J}$ Gastroenterol 13: 5261-5266, 2007.

7. Siriwardena BS, Kudo Y, Ogawa I, Kitagawa M, Kitajima S, Hatano H, Tilakaratne WM, Miyauchi M and Takata T: Periostin is frequently overexpressed and enhances invasion and angiogenesis in oral cancer. Br J Cancer 95: 1396-1403, 2006.

8. Shao R, Bao S, Bai X, Blanchette C, Anderson RM, Dang T, Gishizky ML, Marks JR and Wang XF: Acquired expression of Periostin by human breast cancers promotes tumor angiogenesis through up-regulation of vascular endothelial growth factor receptor 2 expression. Mol Cell Biol 24: 3992-4003, 2004.

9. Yan W and Shao R: Transduction of a mesenchyme-specific gene periostin into $293 \mathrm{~T}$ cells induces cell invasive activity through epithelial-mesenchymal transformation. J Biol Chem 281 19700-19708, 2006
10. Hecht SS: Cigarette smoking and lung cancer: chemical mechanisms and approaches to prevention. Lancet Oncol 3: 461-469, 2002.

11. Dasgupta P, Rizwani W, Pillai S, Kinkade R, Kovacs M, Rastogi S, Banerjee S, Carless M, Kim E, Coppola D, et al: Nicotine induces cell proliferation, invasion and epithelialmesenchymal transition in a variety of human cancer cell lines. Int J Cancer 124: 36-45, 2009.

12. Hong L, Sun H, Lv X, Yang D, Zhang J and Shi Y: Expression of periostin in the serum of NSCLC and its function on proliferation and migration of human lung adenocarcinoma cell line (A549) in vitro. Mol Biol Rep 37: 2285-2293, 2010.

13. Sasaki H, Dai M, Auclair D, Fukai I, Kiriyama M, Yamakawa Y, Fujii Y and Chen LB: Serum level of the periostin, a homologue of an insect cell adhesion molecule, as a prognostic marker in nonsmall cell lung carcinomas. Cancer 92: 843-848, 2001.

14. Sasaki H, Lo KM, Chen LB, Auclair D, Nakashima Y, Moriyama S, Fukai I, Tam C, Loda M and Fujii Y: Expression of Periostin, homologous with an insect cell adhesion molecule, as a prognostic marker in non-small cell lung cancers. Jpn J Cancer Res 92: 869-873, 2001.

15. Ruan K, Bao S and Ouyang G: The multifaceted role of periostin in tumorigenesis. Cell Mol Life Sci 66: 2219-2230, 2009.

16. Cucina A, Dinicola S, Coluccia P, Proietti S, D'Anselmi F, Pasqualato A and Bizzarri M: Nicotine stimulates proliferation and inhibits apoptosis in colon cancer cell lines through activation of survival pathways. J Surg Res: Mar 10, 2012 (Epub ahead of print).

17. Do NY and Lim SC: A low level of nicotine-induced chemoresistance in a KB cell line. Mol Med Report 1: 55-60, 2008.

18. Chen RJ, Ho YS, Guo HR and Wang YJ: Long-term nicotine exposure-induced chemoresistance is mediated by activation of Stat 3 and downregulation of ERK1/2 via nAChR and betaadrenoceptors in human bladder cancer cells. Toxicol Sci 115: 118-130, 2010.

19. Martínez-García E, Irigoyen M, González-Moreno O, Corrales L, Teijeira A, Salvo E and Rouzaut A: Repetitive nicotine exposure leads to a more malignant and metastasis-prone phenotype of SCLC: a molecular insight into the importance of quitting smoking during treatment. Toxicol Sci 116: 467-476, 2010.

20. Xu L and Deng X:Protein kinase Ciota promotes nicotine-induced migration and invasion of cancer cells via phosphorylation of micro- and m-calpains. J Biol Chem 281: 4457-4466, 2006.

21. Cooke JP: Angiogenesis and the role of the endothelial nicotinic acetylcholine receptor. Life Sci 80: 2347-2351, 2007.

22. Kanda Y and Watanabe Y: Nicotine-induced vascular endothelial growth factor release via the EGFR-ERK pathway in rat vascular smooth muscle cells. Life Sci 80: 1409-1414, 2007.

23. Huber MA, Kraut N and Beug H: Molecular requirements for epithelial-mesenchymal transition during tumor progression. Curr Opin Cell Biol 17: 548-558, 2005.

24. Gotti C and Clementi F: Neuronal nicotinic receptors: from structure to pathology. Prog Neurobiol 74: 363-396, 2004 